\title{
CODELAB: A Developers' Tool for Efficient Code Generation and Optimization
}

\author{
Dragan Mirković ${ }^{1}$ and S. Lennart Johnsson ${ }^{1}$ \\ Department of Computer Science \\ University of Houston \\ Houston, TX 77204 \\ mirkovic@cs.uh.edu, johnsson@cs.uh.edu
}

\begin{abstract}
In this paper we describe CODELAB, an integrated development environment (IDE) for efficient code development and optimization. The main idea behind the CODELAB IDE is to bring together several useful code development and optimization tools into an integrated and user friendly environment with a goal of simplifying the production and maintenance of high performance software libraries. We give an overview of the tool structure and provide examples that illustrate the use and the efficiency of the IDE.
\end{abstract}

\section{Introduction}

Over the last 30 years, microprocessors have enjoyed a steady increase in performance inherent in Moore's law. The complexity of microprocessor architectures, however, has also been steadily increasing and the efficient application of scientific computing techniques requires constantly growing amounts of specialized knowledge in a variety of diverse scientific areas such as numerical analysis, algorithm theory, computer architectures, and programming languages. These requirements have put the high performance code development out of reach for many researches, since in most cases, simple implementations achieve only a small fraction of the peak performance on any given advanced architecture.

Most of the scientific software today is written in high-level programming languages and relies on efficient programming methods, good optimizing compilers, and use of hardware-tuned software libraries to achieve high performance. A number of other tools have been developed either to simplify the code writing or to help the optimization. The most popular are scripting (or Very High Level) languages (VHLLs) like MATLAB, Python, or Tcl/Tk. The primary advantage of these languages over a third generation language like $\mathrm{C}$ or $\mathrm{C}++$ is that fewer lines of code must be written to complete a given task. They provide the user with a clear and simple expression of complex computational algorithms and allow for easy construction of graphical user interfaces. A further increase in productivity arises from the fact that scripting languages are usually interpreted which removes the compile-link-run cycle delays. The performance of applications developed with VHLLs, however, strongly depends on the granularity of 
the code and on the quality of numerical and graphical libraries hidden behind the interpreter function calls. In general, long loops with a large number of simple numerical or graphical instructions perform poorly due to high interpreter overhead. To improve the performance in these situations most VHLLs need to be extended by adding procedures written in some lower level language. In many cases use of tightly-integrated tools can cut down the development time. Current trend is to join together compilers, libraries and supporting tools into a single, integrated development environment (IDE), allowing the user creating, compiling, linking, assembling and debugging within a single application.

Even with all these tools one problem still remains. Someone has to update the libraries and optimize them for the hardware that changes very rapidly. The advanced optimization techniques like loop overhead reductions through unrolling, cache optimizations, instruction level parallelism and use of special vector instructions produce code that is very difficult to maintain. The problem is further exacerbated if the library is to be portable since most of these optimizations are platform specific. The expensive and time consuming nature of these optimizations makes them nearly impossible to do by hand and new approaches are needed to address this problem. The current trend to achieve portable performance for scientific software libraries is to design software that can automatically adapt to different architectures either during the installation, or at runtime, or both. Examples of this approach include linear algebra libraries like ATLAS [1] or PHiPAC [2], fast Fourier transform libraries like FFTW [3], UHFFT $[8,9,10]$ or Signal Processing libraries like SPIRAL [13]. These packages usually provide many ways of doing required operations, and use empirical timings or performance estimates in order to choose the best method for a given architecture. The implementation of different ways of performing the same operation can, in some cases be parametrized and the same code can be used on different architectures with parameters selected either at installation/compile time or at run time. More often the only way is to have different source code implementations for the same operation and select the one that performs the best on the given underlaying architecture. These multiple sources can either be hand-written and tuned or automatically generated, where the automatic code generation has a significant advantage from the developers point of view.

There are very few general tools for library code development and most of them are developed by the architecture vendors and are useful only for a restricted set of architectures. These include performance measuring tools and simulators that can efficiently track the flow of instructions and data through the maze of memory hierarchies and instruction pipelines of modern microprocessors and code generators that can produce tens of thousands of lines of computer code in seconds, visualize the code structure and automatically perform sophisticated optimizations. The low level performance measuring tools are hardware-specific and there are very few portable solutions, like PAPI [11] or PCL [12].

In this paper we describe an IDE that joins together several code development tools into a single application with the goal to simplify development and tuning of modern adaptive software libraries. 


\section{Overview of the CODELAB Structure}

The purpose of the CODELAB project is to create a general code generation/performance measurement tool that would enable advanced code developers to easily generate, modify and test the performance of large amounts of code. The CODELAB project is a generalization of our effort in development of an adaptive FFT library $[8,9,10]$ and as such it has inherited many of the tools developed in the UHFFT project. In UHFFT we have developed an efficient automatic method of generating and optimizing the FFT kernels. The code generator used in the UHFFT is based on the abstraction of the numerical algorithm and most of the manipulations and optimizations are done using a general abstract expression algebra. This approach can can be easily applied to other similar, numerical algorithms.

The schematic structure of the CODELAB IDE is shown in Figure 1.

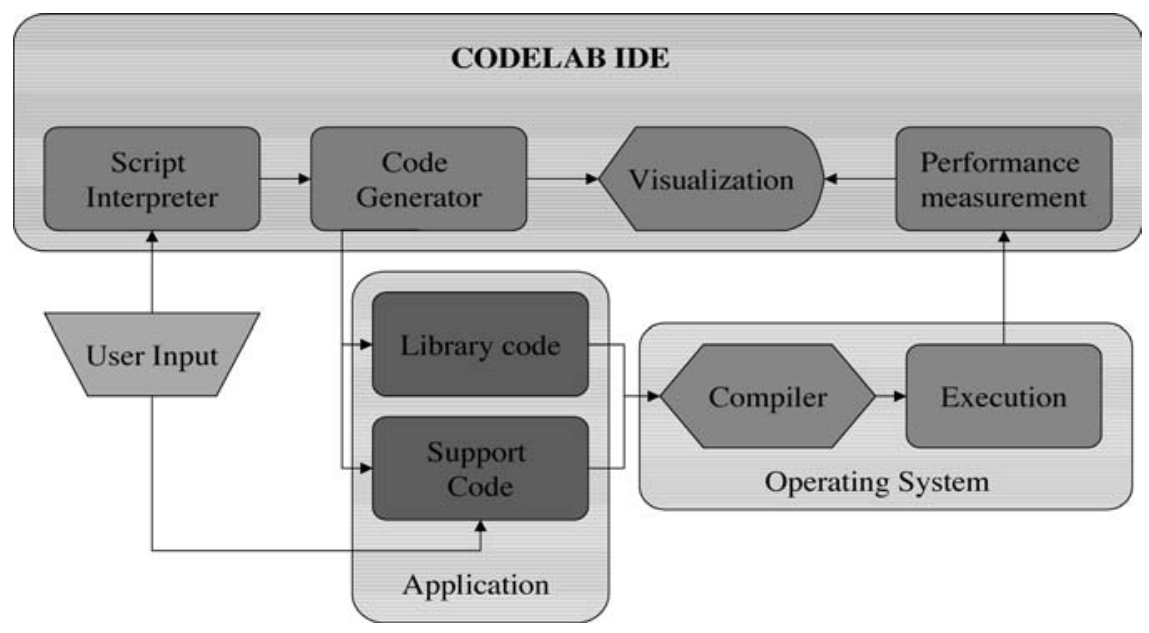

Fig. 1. High-level structural overview of the CODELAB integrated development environment.

\subsection{Applications Suitable for the CODELAB Environment}

The basic assumption in CODELAB is that the application consists of two principal parts. The first part is the library code which contains a large number of highly optimized computational kernels needed in the application. We assume that the kernel algorithm can be parametrized by a small set of parameters like kernel size, dimension, precision, order, blocking factor, factorization options, 
etc. By varying these parameters the same code generator code can produce a wide variety of computational modules.

The second part is the supporting code that binds the library routines together such that the function required by an application can be computed. This part can be either hand-written or automatically generated. The supporting code also contains an appropriate search method which automates the selection of the most optimal available combination of computational modules from the associated library.

There are many important applications that satisfy the requirement of the CODELAB environment and we list here some of them

- Various numerical linear algebra routines. Most of the linear algebra algorithms can be blocked in many different ways and the blocking factor has a strong influence on cache utilization and on the performance of the implementation.

- Discrete Fourier and other linear transforms that can be factored or decomposed in order to obtain a fast algorithm by using a divide-and-conquer approach. These factorizations are not unique, and there are, in general, many different ways to compute the same transform. Here different factorizations not only change the blocking of the algorithm, but may also have a different number of floating point operations.

- Finite element methods (FEM). We are developing a general Lagrangian finite element code generator that can produce a finite element bases of arbitrary order and dimension and a variety of associated element and macroelement stiffness matrices. For a given FEM discretization the order of the basis and the size of the macroelement (i.e., a connected collection of elements) define the blocking factor and the cache utilization.

- Multiprecision calculations. A code generation can be efficiently used to implement a high precision mathematical library with a fixed length of the mantissa. The length of the mantissa can be implemented as a parameter in the code generator and a whole library implementing a variety of mathematical functions can be generated in a short amount of time.

- Various signal and image processing kernels can be implemented as a combination of fast Fourier transform and linear algebra algorithms.

\subsection{The Scripting Language}

The first step in library code development is efficient and simple algorithm descriptions. For this purpose we use a simple scripting language with constructs for standard algorithm elements, like variables, constants, expressions, repetitions and branching, real and complex arithmetic, matrix and vector algebra, trees, graphs, and lists.

The main purpose of the scripting language is to simplify construction of different code generators. In general, a code generator for a given algorithm can be written directly in C, and the script part can be bypassed. Scripts, however, provide for a more compact description of different algorithms. 
The user provides a script for the code generator and some of the supporting code for the application. The code generator produces a library of computational kernels and a portion of the support code. The computational kernels contain, in most of the cases, optimized straight line code.

\subsection{The Code Generator}

The script which describes the algorithm is either interpreted or translated into the code generator which can produce a large variety of computational kernels depending on the input parameters. The first step in code generation is the algorithm abstraction in which a portion of the application algorithm is transformed into a list of expression structures. Hence, the basic structure in the code generator is called Expression. An expression is a polymorphic structure that takes one of the following forms:

- Constant Expression: A constant can be a real constant (REALCONST), an integer constant (INTEGERCONST), a Zero constant(ZEROCONST) or a constant with a value equal to one (ONECONST). All constants are stored as doubles and additional information are used in order to facilitate simplifications of expressions.

- Variable Expression: This is an abstraction for the variables used in different algorithms. The interpretation of the variable depends on its type, which is a composition of two attributes:

o relevance to the algorithm, i.e., input, output or temporary.

o type of data stored, i.e., integer, real, imaginary or complex.

- Sum Expression: This is an abstraction for composite expressions of the form $\sum_{i=0}^{N} e_{i}$, where $e_{i}$ are expressions.

- Product Expression: This is an abstraction for composite expressions of the form $\prod_{i=0}^{N} e_{i}$, where $e_{i}$ are expressions.

- Negative Expression: This is an abstraction for the change of sign. It is also used for subtraction, e.g, the statement $\operatorname{SumExpr}(\operatorname{expr} 1, \operatorname{NegExpr}(\operatorname{Expr} 2))$ amounts to subtracting expr2 from expr1.

- Assign Expression: This is an abstraction for expressions of the form $v=e$ where $v$ is a variable and $e$ is an expression.

- Complex Expression: This is the abstraction for complex arithmetic. it consists of two expressions, one for the real part and one for the imaginary part.

The four basic operations on Expressions are the sum (SumExpr), the product (MultExpr), the quotient (DivExpr), and the sign change (NegExpr). These four operations are used in the generation of Expression Lists. The derived expressions may contain some redundancies which are removed to reduce the total number of arithmetic operations in the expression list. Some of the simplifications include:

- Cancellation of terms in sums, e.g, $(t m p 3+t m p 5)+(t m p 6-t m p 3)$ would yield $t m p 5+t m p 6$. 
- Trivial multiplications are eliminated, i.e., multiplications by $\pm 1, \pm i$ and 0 .

- Common constants are factored out, e.g., $C 2 * t m p 5+C 2 * t m p 7$ would yield $C 2 *(t m p 5+t m p 7)$.

- Constants are folded, e.g., $C 2 * C 3 * C 4 * t m p 5$ would yield $C 5 * t m p 5$, where $C 5=C 2 * C 3 * C 4$.

- Multiple sign changes are simplified, e.g., -(-tmp2) would yield tmp2.

- Complete complex algebra is included, i.e., the generator is able to deal with complex expressions.

From the basic structures described above, other more complex structures are derived. The Expression Vector (ExprVec) is a vector of expressions. Each expression may be of one of the defined types for an expression. The Expression Matrix (ExprMat) is a two dimensional structure of expressions. There are a number of operations that are used to manipulate these structures. These operations obey the rules of vector addition and multiplication, e.g., to add two vectors together, they must be of the same size.

The code generator can also provide information about the number of arithmetic operations in the generated code, efficiency of simplifications that are performed, number of registers or temporary variables needed for execution. The abstraction of the kernel algorithm results in a list of expressions and the structure of the generated code can be visualized either by printing the list of expressions or by plotting the direct acyclic graph (DAG) of the algorithm, see Figure 2. The application code is subsequently compiled and executed and the performance measurements can be collected and visualized. There are several advantages of the automatic code generation in library code development. The code that is optimized for modern architectures is, in general, difficult to write since it may contain large portions of completely unrolled loops (straight line code). If these are generated automatically the developer is relieved of a tedious and error prone task of writing them by hand. Moreover, the code generation introduces an additional level of abstraction and allows for separation of the algorithm description from the algorithm implementation. This means that the same algorithm once described in the code generator scripting language can have a number of different instances of implementation, depending on the various options selected during the optimization and scheduling phase.

Example: FFT code generator As an example, we describe the structure of the code generator for fast Fourier transforms used for fast evaluation of the Discrete Fourier Transform (DFT), which is a matrix-vector product of the form $\mathbf{y}=W_{n} \mathbf{x}$, where $W_{n}=\left(w_{l k}\right)_{l, k=0}^{n-1}$ is the DFT matrix with elements $w_{l k}=\omega_{n}^{l k}$, and $\omega_{n}=e^{-2 \pi i / n}$ is the principal $n$th root of unity. The fast evaluation is obtained through factorization of $W_{n}$ into the product of $O(\log n)$ factors

$$
W_{n} \mathbf{x}=\left(A_{1} A_{2} \ldots A_{r}\right) \mathbf{x},
$$

where the matrices $A_{i}$ are sparse and $A_{i} \mathbf{x}$ involves $O(n)$ arithmetic operations. The factorization (1) for a given $n$ is not unique, and possible variations may 
$\operatorname{tmp0i}=\operatorname{Im}(x[0])+\operatorname{Im}(x[i s])$;

tmp0r $=\operatorname{Re}(x[0])+\operatorname{Re}(x[i s])$;

$\operatorname{tmp} 1 i=\operatorname{Im}(x[0])-\operatorname{Im}(x[i s])$;

tmp1r $=\operatorname{Re}(x[0])-\operatorname{Re}(x[i s])$;

$\operatorname{Re}(y[0])=t m p 0 r ;$

$\operatorname{Im}(y[0])=t m p 0 i ;$

$\operatorname{Re}(y[o s])=t m p 1 r ;$

$\operatorname{Im}(y[o s])=\operatorname{tmp} 1 i$;

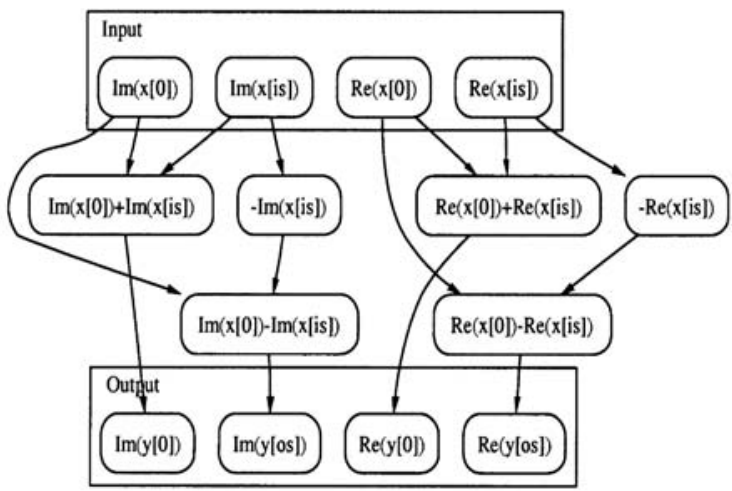

Fig. 2. The list of expressions for the size 2 complex-to-complex fft and the corresponding DAG.

have properties that are substantially different. The code generator used for building the library of FFT kernels in our example uses mixed-radix, prime factor, split-radix, and Rader's algorithms. (see [5, 6, 10] for a more detailed description of these algorithms). The FFT code generator is implemented using a fixed set of factorization rules in a recursive fashion. An additional layer of software has been added to the code generator library to facilitate building of expression lists related to the fast Fourier transforms. They include:

- GenDFTExprMat: this function returns the DFT expression matrix that consists of complex constant expressions. It takes in the DFT size and the transform direction as its input arguments.

- GenFFTExprList: this function generates the expression list for the FFT of size $n$. It takes in as arguments the input vector (to be transformed) and the direction of the required transform and then calls the appropriate FFT algorithm, depending on the size of the input vector. Pseudo code for this functions is as follows:

\section{GenFFTExprList ( ExprVec $* u)$}

$n=$ Length of vector $\mathbf{u}$

if $n=2 \quad$ apply DFT to the vector $\mathbf{u}$

else if $n$ is prime call RADER'S algorithm

else \{ choose factor $r$ of $n$

if $r$ and $n / r$ are co-prime call PRIME FACTOR algorithm else if $n>8$ and $n \bmod 4=0$ call SPLIT-RADIX algorithm else call MIXED-RADIX algorithm 
- IxFFT $(r, m)$ : this function evaluates the tensor (kroneker) product of the identity matrix of size $r$ with the FFTExprList of size $m$. This operation is also known as the parallel FFT.

- FFTxI $(r, m)$ : this function evaluates the tensor (kroneker) product of the FFTExprList of size $r$ with the identity matrix of size $m$. This operation is also known as the vector FFT.

- Mixed-Radix Algorithm: This returns a list of expressions which is an abstraction for the Mixed-Radix FFT algorithm.

- Split-Radix Algorithm: This returns a list of expressions which is an abstraction for the Split-Radix FFT algorithm.

- Prime Factor Algorithm: This returns a list of expressions which is an abstraction for the Prime Factor FFT algorithm.

- Rader's Algorithm: This returns a list of expressions which is an abstraction for Rader's FFT algorithm.

\subsection{Performance Measurements and Tools}

The CODELAB IDE has an integrated infrastructure for detailed performance measurements. These measurements provide a valuable feedback about critical points of the generated code and point to ways of improving performance. All of the generated code can be automatically instrumented for simple or advanced performance measurements using hardware performance counters. A simple visualization of the performance is included in the IDE. The low level performance measurements are implemented as an interface to the PAPI library [11].

Some of the specific uses of the performance data in the IDE include:

- Parameter specification for the code generator. One of the assumptions in code generation is that the kernel algorithm can be parametrized by a small set of parameters. Some of these parameters, like blocking factors or factorization options, are performance related and performance information is needed in the selection process.

- Building of performance models for CODELAB applications. Many adaptive applications make use of either direct performance measurements or need a performance model as a part of the application. Easy access to the performance data is essential in building a performance model.

- Interactive code optimization. Some of the code optimizations have to be done manually and this is usually a very tedious and error prone task. Interactive access to the performance data provided in the CODLAB IDE can alleviate some of the difficulties in this process.

As an example, in Figure 3 we show performance maps for some of the codelets from the FFT library. These are given with respect to input and output strides. The collection of codelets constitutes the core of the FFT library and most of the transform CPU time is spent executing the codelets that constitute the transform plan. Each codelet, smaller in size than the whole transform, acts on a subset of data defined by the input stride. The output vector is also, 

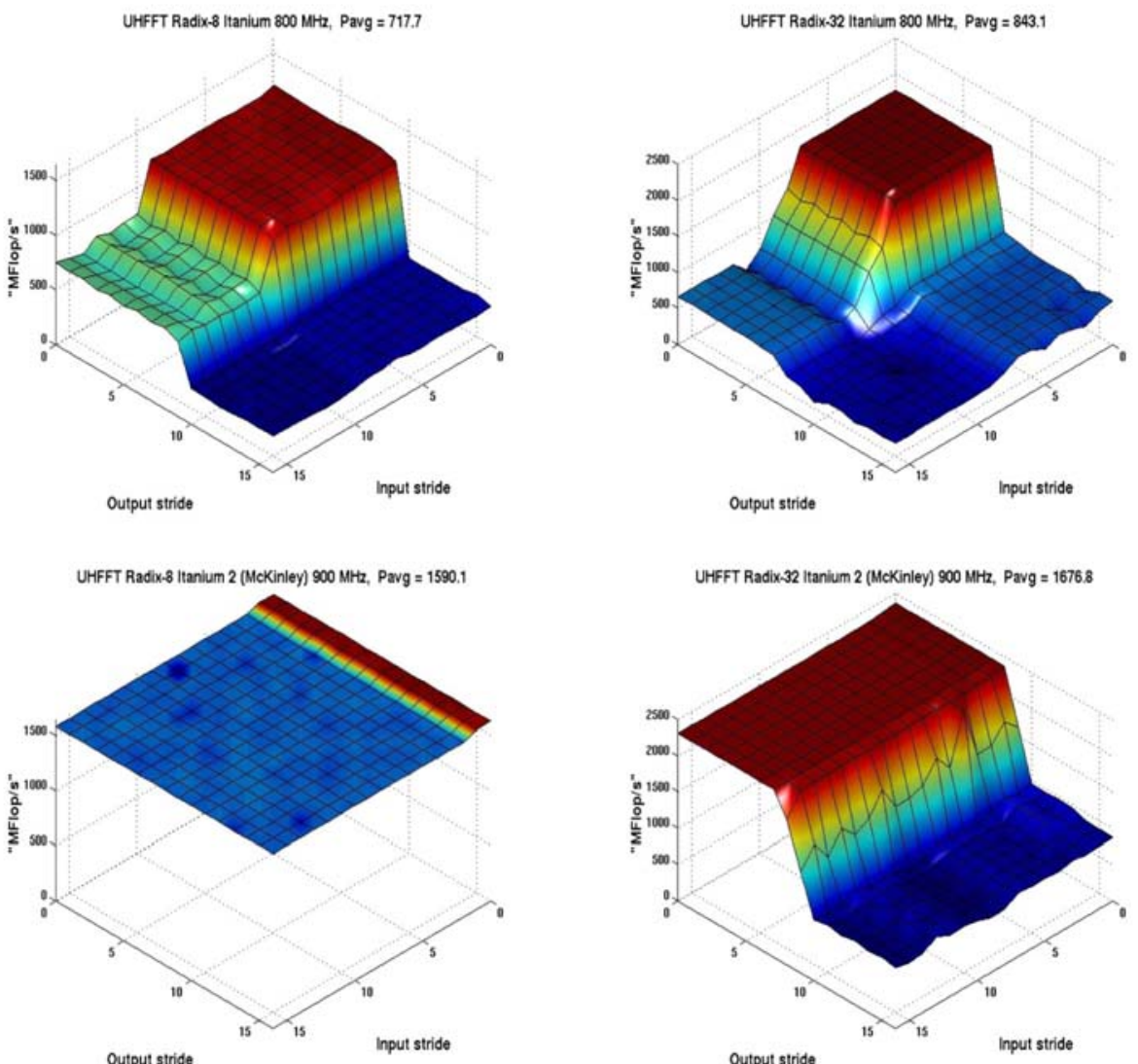

UHFFT Radix-8 UltraSpare $111750 \mathrm{MH}$, Pavg $=349.7$

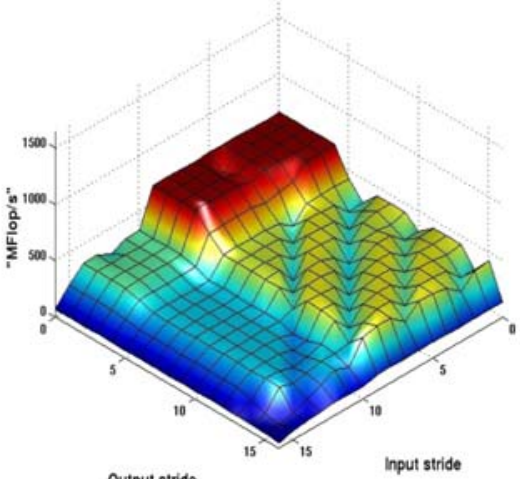

Output stride

Input stride

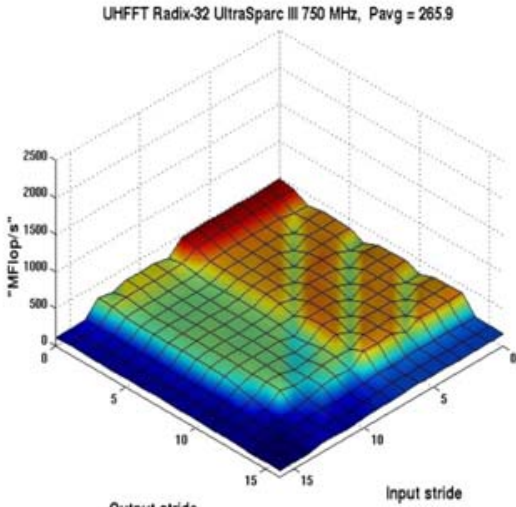

Output stride

Fig- 3. Performance maps for codelets with sizes $n=8$ and $n=32$ on the $800 \mathrm{MHz}$ Intel Itanium, $900 \mathrm{MHz}$ Intel Itanium 2, and $750 \mathrm{MHz}$ S1ur UltraSparc-III. 
in general, accessed in a strided fashion. For that reason, strided performance of codelets is key to understanding the overall performance of the library. The UHFFT library uses a performance model for the codelets to speed up the selection of the optimal plan for the specific transform and this performance model is based on the data shown in Figure 3.

Acknowledgment.

This work has been carried out with partial support from the DOE funded Los Alamos Computer Science Institute, LACSI, the GrADS projected funded under the Next Generation Software initiative with the National Science Foundation, and in part by the Alliance funded as part of the NSF PACI program.

\section{References}

[1] Whaley, R., Petitet, A., Dongarra, J. Automated Empirical Optimization of Software and the ATLAS Project. Parallel Computing, 27:3-25, 2001.

2] J. Bilmes and K. Asanović and J. Demmel and D. Lam and C.W. Chin. The PHiPAC WWW Home Page http://www.icsi.berkeley.edu/ ${ }^{\text {bilmes/phipac }}$

[3] Matteo Frigo and Steven G. Johnson. The Fastest Fourier Transform in the West. Technical Report MIT-LCS-TR-728, MIT, 1997.

[4] Charles Van Loan. Computational frameworks for the fast Fourier transform. Philadelphia:SIAM, 1992.

[5] Richard Tolimieri, Myoung An, and Chao Lu. Algorithms for Discrete Fourier Transforms and Convolution. Springer-Verlag, New York, 1 edition, 1989.

[6] P. Duhamel and M. Vetterli. Fast Fourier Transforms: A Tutorial Review and a State of the Art. Signal Processing, 19:259-299, 1990.

[7] Matteo Frigo. A Fast Fourier Transform Compiler. Proceedings of the 1999 ACM SIGPLAN Conference on Programming Language Design and Implementation, pages $169-180,1999$.

[8] Dragan Mirković, Rishad Mahasoom, and Lennart Johnsson. An Adaptive Software Library for Fast Fourier Transforms. Proceedings of the 2000 International Conference on Supercomputing, pages 215-224, 2000.

[9] Dragan Mirković and Lennart Johnsson. Automatic Performance Tuning in UHFFT Library. Proceedings of the 2001 International Conference on Computational Science, Lecture Notes in Computer Science 2073, Vol. 1, pages. 71-80, 2001.

[10] Dragan Mirković and Lennart Johnsson. Automatic Performance Tuning for Fast Fourier Transforms. International Journal of High Performance Computing Applications, to appear.

[11] The PAPI web page. http://icl.cs.utk.edu/projects/papi/.

[12] The Performance Counter Library web page. ww. fz-juelich.de/zam/PCL/.

[13] Home Page of the SPIRAL Project. http://www.ece.cmu.edu/ "spiral. 